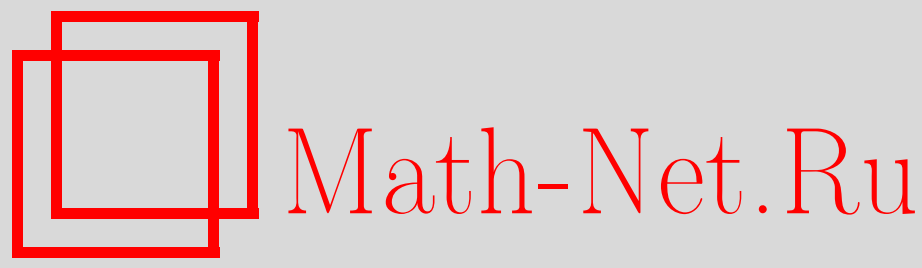

А. И. Бреев, Поляризация вакуума скалярного поля на однородных пространствах с инвариантной метрикой, TMФ, 2014, том 178, номер 1, 69-87

DOI: https://doi.org/10.4213/tmf8511

Использование Общероссийского математического портала Math-Net.Ru подразумевает, что вы прочитали и согласны с пользовательским соглашением http://www . mathnet.ru/rus/agreement

Параметры загрузки:

IP : 54.162 .127 .20

26 апреля 2023 г., 06:18:28

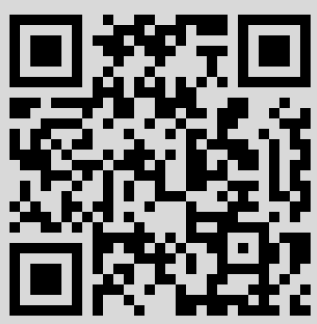




\title{
ПОЛЯРИЗАЦИЯ ВАКУУМА СКАЛЯРНОГО ПОЛЯ НА ОДНОРОДНЫХ ПРОСТРАНСТВАХ С ИНВАРИАНТНОЙ МЕТРИКОЙ
}

\begin{abstract}
Разработан метод вычисления вакуумных средних тензора энергии-импульса скалярного поля на однородных пространствах с инвариантной метрикой. Для решения поставленной задачи используется метод обобщенного гармонического анализа, базирующийся на методе орбит коприсоединенного представления.
\end{abstract}

Ключевые слова: поляризация вакуума, тензор энергии-импульса, гармонический анализ на однородных пространствах.

DOI: $10.4213 / \operatorname{tmf} 8511$

\section{1. ВВЕДЕНИЕ}

Квантовая теория поля в искривленном пространстве-времени является достаточно разработанной теорией (см. монографии [1]-[3] и обзор [4]), представляющей интерес в связи с актуальными приложениями к космологии и астрофизике. Наиболее важной величиной, характеризующей материю, является тензор энергии-импульса (ТЭИ). Он играет роль источника гравитационного поля в уравнениях Эйнштейна и описывает взаимодействие материи с гравитационным полем. Средние значения ТЭИ по вакуумному состоянию характеризуют эффект поляризации вакуума и, если вакуумное состояние определено неоднозначно, то также эффекты рождения частиц гравитационным полем.

При вычислении квантовых средних от билинейных по полю операторов (в том числе от ТЭИ) по любому состоянию появляются расходимости, так как билинейные операторы содержат произведения операторнозначных обобщенных функций. Поэтому для получения конечных значений вакуумных средних ТЭИ необходимо использовать некоторую процедуру устранения расходимостей. В случае, когда пространство однородно и изотропно, эффективно применение метода размерной

*Национальный исследовательский Томский государственный университет, Томск, Россия. E-mail: breev@mail.tsu.ru

${ }^{\dagger}$ Национальный исследовательский Томский политехнический университет, Томск, Россия 
регуляризации [5]. Другой способ регуляризации - метод раздвижения аргументов операторов поля в билинейной форме ТЭИ, предложенный в работе [6]. Стоит отметить, что хотя упомянутые выше методы регуляризации не требуют расчета вакуумных средних ТЭИ, расчет последних также имеет значение, так как в ряде случаев возможно избавиться от расходимости напрямую (например, при помощи метода $n$-волновой регуляризации [7]).

Отметим, что практически все известные в настоящее время модели римановых многообразий общей теории относительности связаны с различными группами преобразований и нередко относятся к классу однородных римановых пространств. В современной космологии на базе однородных пространств строятся модели Большого взрыва, начальных сингулярностей, инфляционные модели. Естественно возникает задача учета квантовых вакуумных эффектов на однородных пространствах.

С данной задачей тесно связана проблема точного интегрирования релятивистских волновых уравнений на многообразиях с кривизной и нетривиальной топологией. Наиболее универсальным методом решения является метод разделения переменных (МРП) [8], [9]. Но МРП учитывает только коммутативную алгебру симметрии уравнения, и существует класс пространств, не допускающих разделения переменных. Поэтому в большинстве случаев расчет вакуумных квантовых эффектов возможно провести, налагая на метрику пространства различные ограничения (например, конформно-плоские метрики [2], однородные изотропные пространства [1], штеккелевы метрики [10] и т. д.), позволяющие проинтегрировать волновые уравнения поля.

Настоящая работа посвящена вычислению вакуумных средних ТЭИ скалярного поля на однородных пространствах с произвольной инвариантной метрикой статического пространства-времени.

Для решения поставленной задачи применяется метод орбит, который позволяет провести некоммутативную редукцию (метод некоммутативного интегрирования [11], [12]) волнового уравнения Клейна-Гордона к уравнению с меньшим количеством независимых переменных, на многообразии, обладающем более простой геометрией и топологией. Данный метод, в отличие от МРП, учитывает некоммутативную алгебру симметрии уравнения Клейна-Гордона. Причем решение строится глобально и не зависит от выбора локальных координат на однородном пространстве. Метод орбит коприсоединенного представления (К-орбит) впервые описан в работах Кириллова [13], [14] и развит в последующих работах Кириллова, Константа, Сурье и др. Основные результаты метода приведены в монографиях [15]-[17].

В предыдущей работе авторов [18] при помощи метода орбит исследовались частные случаи, когда однородное пространство коммутативно (дефект однородного пространства равен нулю) и когда оно является группой Ли (случай, когда дефект максимален). В настоящей работе при интегрировании волнового уравнения учитывается алгебра инвариантных операторов на однородном пространстве и дефект пространства предполагается произвольным.

В разделах 2, 3 кратко излагаются метод К-орбит и основанный на нем гармонический анализ на однородных пространствах. Более подробное изложение с доказательствами основных утверждений можно найти в работах [19]-[22].

Раздел 4 посвящен использованию метода орбит для некоммутативной редукции уравнения Клейна-Гордона. Получены соотношения, выражающие базис решений 
уравнения Клейна-Гордона через базис решений редуцированного уравнения и удовлетворяющие условию нормировки скалярного поля. Заметим, что для проведения некоммутативной редукции на однородном пространстве, обладающем ненулевым дефектом, существенно используется алгебра инвариантных операторов (в дальнейшем $\mathcal{F}$-алгебра) на однородном пространстве. Найдено выражение для обобщенной локальной дзета-функции оператора уравнения Клейна-Гордона и показано, что оно не зависит от выбора локальных координат на однородном пространстве, а определяется через величины, определенные на лагранжевом подмногообразии к симплектическому листу $\mathcal{F}$-алгебры. Это позволяет облегчить нахождение аналитического продолжения дзета-функции в конкретных задачах, так как выражение для дзета-функции получается более простым и зависит от меньшего количества независимых переменных.

В разделе 5 рассматривается ТЭИ скалярного поля в статическом пространствевремени. ТЭИ рассматривается в квазитетрадных компонентах, введенных в работе [18]. Такой подход позволяет обойтись без использования локальных координат на однородном пространстве, причем в конце вычислений всегда можно перейти к обычным компонентам ТЭИ. Найдены выражения для вакуумных средних ТЭИ в квазитетрадных компонентах, которые определяются алгебраическими характеристиками однородного пространства (такими как $\lambda$-представление алгебры Ли группы Ли преобразований и $\mathcal{F}$-алгебры инвариантных операторов) и не зависят от локальных координат на однородном пространстве. Для получения конечных значений вакуумных средних ТЭИ применяется модификация метода обобщенной дзета-функции, предложенная в работе [23]. В работе [18] метод обобщенной функции применялся для вычисления вакуумных средних ТЭИ на группах Ли с инвариантной метрикой. Данный метод перенормировки вакуумных средних ТЭИ основан на вычислении функциональных производных от однопетлевого эффективного действия по метрике и сводит задачу к поиску аналитического продолжения для обобщенной дзета-функции.

В качестве нетривиального примера рассмотрено однородное пространство с дефектом, равным единице, и четырехмерной алгеброй Ли преобразований, являющейся прямым произведением абелевой двумерной алгебры и двух одномерных идеалов. Для произвольной инвариантной метрики статического пространства-времени найдены выражение для обобщенной локальной дзета-функции и перенормированное значение плотности энергии вакуума скалярного поля, обусловленного нетривиальной топологией и кривизной данного однородного пространства.

\section{2. $\lambda$-ПРЕДСТАВЛЕНИЕ АЛГЕБРЫ ЛИ И ГРУППЫ ЛИ НА К-ОРБИТЕ}

Пусть $G$ - связная односвязная вещественная группа Ли, $\mathfrak{g}$ - алгебра Ли группы Ли $G, \mathfrak{g}^{*}$ - пространство линейных функционалов на $\mathfrak{g}$. Пусть группа Ли $G$ действует на сопряженном пространстве $\mathfrak{g}^{*}$ коприсоединенным представлением $\mathrm{Ad}^{*}: G \times$ $\mathfrak{g}^{*} \rightarrow \mathfrak{g}^{*}$. Данное действие расслаивает $\mathfrak{g}^{*}$ на K-орбиты размерности $\operatorname{dim} \mathfrak{g}-$ ind $\mathfrak{g}-2 k$, где число $k$ принимает значения от 0 до $(\operatorname{dim} \mathfrak{g}-$ ind $\mathfrak{g}) / 2$. Индекс алгебры ind $\mathfrak{g}$ определяется как количество независимых функций Казимира на сопряженном пространстве $\mathfrak{g}^{*}$ относительно скобки Пуассона-Ли $\{\cdot, \cdot\}^{\mathrm{Lie}}$. В работе [20] показано, что 
коалгебра $\mathfrak{g}^{*}$ является объединением связных инвариантных алгебраических поверхностей $M_{(s)}$, где каждая связная поверхность $M_{(s)}$ является объединением К-орбит размерности $\operatorname{dim} \mathfrak{g}-$ ind $\mathfrak{g}-2 s$.

В дальнейшем мы будем использовать терминологию, введенную в работах [19], [20]. Непостоянные на $M_{(s)}$ функции $K_{\mu}^{(s)}(f)$, коммутирующие с любой функцией на $M_{(s)}$, называются функциями Казимира $(s)$-типа. Число $r_{(s)}$ функционально независимых функций Казимира $(s)$-типа совпадает с размерностью пространства $r_{(s)}=\operatorname{dim} M_{(s)}$. K-орбита называется орбитой $s$-типа, если $\mathcal{O}_{\lambda} \in M_{(s)}$, а число $s$ - степенъю вырождения орбиты. К-орбиты с нулевой степенью вырождения называются невырожденными, а остальные сингулярными. Через $F_{\alpha}^{(s)}(f), \alpha=$ $1, \ldots, \operatorname{dim} \mathfrak{g}-r_{(s)}$, обозначим независимый набор функций, определяющих поверхность $M_{(s)}$.

Пусть $\mathcal{O}_{\lambda}-\mathrm{K}$-орбита группы Ли $G(s)$-типа, содержащая ковектор $\lambda$. Форма Кириллова $\omega_{\lambda}$ задает на $\mathrm{K}$-орбите $\mathcal{O}_{\lambda}$ симплектическую структуру. Введем на К-орбите канонические координаты Дарбу $(p, q) \in P \times Q$, в которых форма Кириллова $\omega_{\lambda}$ принимает канонический вид: $\omega_{\lambda}=d p_{a} \wedge d q^{a}, a=1, \ldots, \operatorname{dim} \mathcal{O}_{\lambda} / 2$. Очевидно, что области $P$ и $Q$ являются лагранжевыми подмногообразиями размерности $\operatorname{dim} \mathcal{O}_{\lambda} / 2$. Согласно [22] определим каноническое вложение $f: \mathcal{O}_{\lambda} \rightarrow \mathfrak{g}^{*}$, когда ковектору $f \in \mathfrak{g}^{*}$ ставятся в соответствие его канонические координаты на соответствующей К-орбите. Каноническое вложение однозначно определяется функциями $f_{X}=f_{X}(p, q, \lambda)$, удовлетворяющими системе уравнений

$$
\left\{f_{X}, f_{Y}\right\}^{\text {Lie }}=f_{[X, Y]}, \quad f_{X}(0,0, \lambda)=\lambda(X), \quad X, Y \in \mathfrak{g} .
$$

Так как $f \in M_{(s)}$, то в случае сингулярных К-орбит каноническое вложение также должно удовлетворять условию $F_{\alpha}^{(s)}(f)=0, \alpha=1, \ldots, \operatorname{dim} \mathfrak{g}-r_{(s)}$.

Перейдем от алгебры Ли $\mathfrak{g}$ к соответствующему комплексному расширению $\mathfrak{g}_{\mathbb{C}}$ и рассмотрим каноническое вложение, линейное по переменным $p$ :

$$
f_{X}(q, p, \lambda)=\alpha_{X}^{a}(q) p_{a}+\chi_{X}(q, \lambda), \quad X \in \mathfrak{g}_{\mathbb{C}}, \quad a=1, \ldots, \operatorname{dim} Q .
$$

В работе [22] показано, что для существования линейного канонического вложения (1) орбиты $\mathcal{O}_{\lambda}$ необходимо и достаточно, чтобы функционал $\lambda$ допускал nоляризацию $\mathfrak{p}$. Напомним, что поляризацией $\mathfrak{p}$ функционала $\lambda$ называется подалгебра в $\mathfrak{g}_{\mathbb{C}}$ размерности $\operatorname{dim} \mathfrak{p}=\operatorname{dim} \mathfrak{g}-\operatorname{dim} \mathcal{O}_{\lambda} / 2$, подчиненная функционалу $\lambda:\langle\lambda,[\mathfrak{p}, p]\rangle=0$. Отметим, что поляризация $\mathfrak{p}$ является подалгеброй изотропии алгебры $\mathfrak{g}_{\mathbb{C}}$ локальной группы $G_{\mathbb{C}}$, действующей на локальном однородном пространстве $Q \approx G_{\mathbb{C}} / e^{p}$.

Проведем процедуру квантования К-орбит, которая заключается в сопоставлении каждому спектральному типу орбит специального представления алгебры Ли [20]. Функциям канонического вложения $f_{X}(p, q, \lambda)$ соответствуют операторы $\hat{f}_{X}(q, \lambda)=$ $f_{X}\left(-i \partial_{q}, \hat{q}, \lambda\right)$. Данная процедура квантования однозначна при наложении условия

$$
i\left[\hat{f}_{X}, \hat{f}_{Y}\right]=\hat{f}_{[X, Y]}, \quad X, Y \in \mathfrak{g} .
$$

Операторы $l_{X}(q, \lambda) \equiv i f_{X}(\hat{q}, \hat{p}, \lambda)$ реализуют неприводимое представление алгебры Ли $\mathfrak{g}$ в пространстве гладких функций $L(Q, \mathfrak{p}, \lambda)$ (так называемое $\lambda$-представление алгебры Ли [19], [20]). 
Введем на многообразии $Q$ меру $d \mu_{0}(q)$ и скалярное произведение

$$
\left(\psi_{1}, \psi_{2}\right)=\int_{Q} \overline{\psi_{1}(q)} \psi_{2}(q) d \mu(q), \quad d \mu(q)=\Delta(q) d \mu_{0}(q),
$$

где $\Delta(q)=\Delta\left(s(q), e_{H}\right), \Delta(g)$ - модуль группы Ли $G, s: Q \rightarrow G_{\mathbb{C}}$ - гладкое сечение расслоения $G_{\mathbb{C}}$ с базой $Q$ и слоем $e^{\mathfrak{p}}$. Потребуем, чтобы операторы $\lambda$-представления были косоэрмитовы относительно меры $d \mu_{0}(q)$. Для выполнения этого условия достаточно ввести соответствующий "квантовый сдвиг" на вещественный вектор $\beta$ в операторы $\lambda$-представления: $l_{X}(q, \tilde{\lambda})=l_{X}(q, \lambda+i \beta)$ (см. работу [24]).

Введем поднятие $\lambda$-представления алгебры Ли $\mathfrak{g}$ до локального представления ее группы Ли $G$ :

$$
T^{\lambda}(g) \varphi(q)=\int D_{q \bar{q}^{\prime}}^{\lambda}(g) \varphi\left(q^{\prime}\right) d \mu\left(q^{\prime}\right),\left.\quad \frac{\partial}{\partial t}\right|_{t=0} T^{\lambda}\left(e^{t X}\right) \varphi(q)=l_{X}(q, \lambda) \varphi(q),
$$

где $\varphi \in L(Q, d \mu(q))$. Можно показать (см. [19]), что обобщенные функции $D_{q \bar{q}^{\prime}}^{\lambda}(g)$ удовлетворяют переопределенной системе уравнений

$$
\left[\eta_{X}(g)+l_{X}(q, \lambda)\right] D_{q \bar{q}^{\prime}}^{\lambda}(g)=0, \quad\left[\xi_{X}(g)-\overline{l_{X}^{\dagger}\left(q^{\prime}, \lambda\right)}\right] D_{q \bar{q}^{\prime}}^{\lambda}(g)=0,
$$

где $\xi_{X}(g)=\left(L_{g}\right)_{*} X, \eta_{X}(g)=-\left(R_{g}\right)_{*} X, X \in \mathfrak{g}, g \in G,-$ лево- и правоинвариантные векторные поля на группе Ли $G$. В работе [20] показано, что из требования однозначной определенности функций $D_{q \bar{q}^{\prime}}^{\lambda}(g)$ на группе Ли $G$ следует условие Кириллова целочисленности орбиты $\mathcal{O}_{\lambda}[15]$ :

$$
\frac{1}{2 \pi} \int_{\gamma \in H_{2}\left(\mathcal{O}_{\lambda}\right)} \omega_{\lambda}=n_{\gamma} \in \mathbb{Z} .
$$

Инвариантному подпространству $M_{(s)}$ пространства $\mathfrak{g}^{*}$ сопоставим инвариантное функциональное подпространство $L_{(s)}=\left\{\phi \in L_{2}(G, d \mu(g)) \mid F_{\alpha}^{(s)}(\xi) \phi(g)=0\right\}$ пространства $L_{2}(G, d \mu(g))$. Семейство обобщенных функций $D_{q \bar{q}^{\prime}}^{\lambda}(g)$ обладает свойствами полноты и ортогональности, поэтому для каждой функции $\phi(g)$ из $L_{(s)}$ определены прямое и обратное преобразования Фурье [19]:

$$
\begin{gathered}
\psi\left(q, q^{\prime}, \lambda\right)=\Delta^{-1}(q) \int \phi(g) \overline{D_{q \bar{q}^{\prime}}^{\lambda}}\left(g^{-1}\right) d \mu(g), \\
\phi(g)=\int \psi\left(q, q^{\prime}, \lambda\right) D_{q \bar{q}^{\prime}}^{\lambda}\left(g^{-1}\right) d \mu(q) d \mu\left(q^{\prime}\right) d \mu(\lambda),
\end{gathered}
$$

где $d \mu(\lambda)$ - спектральная мера операторов Казимира $K_{\mu}(\eta)$ на группе, $d \mu(g)$ - правая мера Хаара на группе Ли $G$. Для невырожденных орбит прямое и обратное преобразования (3), (4) определены на всем пространстве $L_{(0)}=L_{2}(G, d \mu(g))$.

\section{3. $\lambda$-ПРЕДСТАВЛЕНИЕ АЛГЕБРЫ ИНВАРИАНТНЫХ ОПЕРАТОРОВ И ГАРМОНИЧЕСКИЙ АНАЛИЗ НА ОДНОРОДНЫХ ПРОСТРАНСТВАХ}

Рассмотрим правое однородное пространство $M$, допускающее группу движений $G$. Любая точка $x \in M$ однородного пространства определяет подгруппу изотропии $H_{x} \in G$, оставляющую данную точку на месте. Пусть $H$ - замкнутая стационарная подгруппа некоторой точки $x_{0} \in M, \mathfrak{h}-$ ее алгебра Ли. Однородное пространство $M$ диффеоморфно фактор-многообразию $G / H$ правых смежных классов 
группы Ли $G$ по подгруппе изотропии $H$, а группу преобразований $G$ можно рассматривать как расслоенное многообразие расслоения $(G, \pi, M, H)$ со структурной группой $H$, базой $M$ и канонической проекцией $\pi: G \rightarrow M$. Линейное пространство алгебры Ли $\mathfrak{g}$ допускает разложение в прямую сумму подпространств $\mathfrak{g}=\mathfrak{h} \oplus \mathfrak{m}$, где $\mathfrak{m} \simeq T_{x_{0}} M-$ дополнение к $\mathfrak{h}$.

Как известно [25], каждой группе Ли $G$, действующей на однородном пространстве $M$, соответствует ассоциативная алгебра $D(M)$ инвариантных дифференциальных и псевдодифференциальных операторов, коммутирующих с генераторами группы.

В алгебре $D(M)$ можно выделить конечный набор функционально независимых образующих $\left\{L_{\mu}\right\}$, которые являются симметрическими функциями от операторов $-i \hbar \eta$ и удовлетворяют нелинейным коммутационным соотношениям

$$
\frac{i}{\hbar}\left[L_{\mu}, L_{\nu}\right]=\Omega_{\mu \nu}(L),
$$

где $\Omega_{\mu \nu}(L)$ - симметрическая функция от операторов $L_{\mu \nu}$. Алгебра с нелинейными коммутационными соотношениями вида (5) называется функционалъной алгеброй $(\mathcal{F}$-алгеброй) [12].

Число $s_{M}=\operatorname{dim} \mathfrak{g}-$ ind $\mathfrak{g}-d_{\max }$, где $d_{\max }-$ максимальная размерность К-орбит $(s)$-типа, имеющих ненулевое пересечение с подпространством $\mathfrak{h}^{\perp}=\left\{f \in \mathfrak{g}^{*} \mid f(X)=\right.$ $0, X \in \mathfrak{h}\}$, называется степенъю вырождения однородного пространства $M$. В работе [21] показано, что набор из $i_{M}$ независимых тождеств (функциональных соотношений между генераторами группы преобразований) на однородном пространстве $M$ состоит из функций $\Gamma(f)=\left\{F_{\alpha}^{\left(s_{M}\right)}(f), \tilde{K}_{\mu}^{\left(s_{M}\right)}(f)\right\}$, где $\tilde{K}_{\mu}^{\left(s_{M}\right)}(f)-$ тривиальные функции Казимира $\left(s_{M}\right)$-типа (тривиальные функции Казимира равны нулю для всех $\left.f \in \mathfrak{h}^{\perp}\right)$. Число $i_{M}$ называется индексом однородного пространства. Индекс и степень вырождения однородного пространства определяются структурными константами алгебры $\mathfrak{g}$ группы преобразований и подалгебры изотропии $\mathfrak{h}[21]$ :

$$
s_{M}=\frac{1}{2} \sup _{\lambda \in \mathfrak{h}^{\perp}} \operatorname{rank}\langle\lambda,[\mathfrak{g}, \mathfrak{g}]\rangle-\frac{1}{2} \operatorname{ind} \mathfrak{g}, \quad i_{M}=\operatorname{dim} \mathfrak{h}-\operatorname{rank}\langle\lambda,[\mathfrak{g}, \mathfrak{h}]\rangle,
$$

где $\lambda$ - элемент общего положения пространства $\mathfrak{h}^{\perp}$. Целое положительное число

$$
d(M)=\operatorname{dim} M+i_{M}-s_{M}-\frac{1}{2}(\operatorname{dim} \mathfrak{g}+\operatorname{ind} \mathfrak{g})
$$

называется дефектом однородного пространства, оно было введено в работах [19], [21]. Дефект однородного пространства характеризует свойства алгебры инвариантных операторов. Для коммутативных пространств $(d(M)=0)$, которыми, в частности, являются симметрические и слабо симметрические пространства, алгебра инвариантных операторов коммутативна и состоит из операторов Казимира $K_{\mu}^{\left(s_{M}\right)}(X)$.

Алгебре инвариантных операторов $D(M)$ соответствует пуассонова алгебра $\mathcal{F}$ с коммутационными соотношениями

$$
\left\{a_{\mu}, a_{\nu}\right\}^{\mathrm{Lie}}=\Omega_{\mu \nu}^{\mathrm{cl}}(a), \quad \Omega_{\mu \nu}^{\mathrm{cl}}(a) \equiv \lim _{\hbar \rightarrow 0} \Omega_{\mu \nu}(a),
$$

где $a_{\mu}(f) \equiv \lim _{\hbar \rightarrow 0} L_{\mu}(f)$ - функция на дуальном пространстве $\mathfrak{g}^{*}$ (символ оператора $\left.L_{\mu}\right)$. Пусть $\mathcal{F}^{*}$ - линейное пространство, сопряженное к $\mathcal{F}$. Бесконечномерное 
линейное пространство гладких функций на $\mathcal{F}^{*}$ является пуассоновой алгеброй относительно скобки Пуассона

$$
\{\varphi(a), \psi(a)\}^{\mathcal{F}} \equiv \Omega_{\mu \nu}^{\mathrm{cl}}(a) \partial^{\mu} \varphi(a) \partial^{\nu} \psi(a), \quad \partial^{\mu} \equiv \frac{\partial}{\partial a_{\mu}}, \quad a \in \mathcal{F}^{*} .
$$

Скобка Пуассона $(7)$ в общем случае является вырожденной, и пространство $\mathcal{F}^{*}$ расслаивается на симплектические листы

$$
O_{\sigma}=\left\{a \in \mathcal{F}^{*} \mid Z_{t}(a)=\sigma_{t}=\text { const }, t=1, \ldots, \text { ind } \mathcal{F}\right\},
$$

где функции $Z_{t}(a)$ называются функииями Казимира $\mathcal{F}$-алгебры и находятся из системы уравнений $\Omega_{\mu \nu}^{\text {cl }}(a) \partial^{\mu} Z_{t}(a)=0$. Число ind $\mathcal{F}$ равно количеству независимых функций Казимира $Z_{t}(a)$ и называется индексом $\mathcal{F}$-алгебры. Размерность и индекс $\mathcal{F}$-алгебры определяются по формулам [21]

$$
\operatorname{dim} \mathcal{F}=i_{M}+2 \operatorname{dim} M-\operatorname{dim} \mathfrak{g}, \quad \text { ind } \mathcal{F}=\operatorname{ind} \mathfrak{g}+2 s_{M}-i_{M}
$$

Размерность симплектического листа $O_{\sigma}$ определяется дефектом однородного пространства: $\operatorname{dim} O_{\sigma}=\operatorname{dim} \mathcal{F}-\operatorname{ind} \mathcal{F}=2 d(M)$.

Заметим, что количество функций Казимира $Z_{t}(a)$ на пространстве $\mathcal{F}^{*}$ совпадает с количеством нетривиальных функций Казимира $K_{\mu}(f)$, и существуют такие функции $D_{t}(Z)$, что

$$
D_{t}(Z(a(f)))=K_{t}^{\left(s_{M}\right)}(f), \quad f \in \mathfrak{h}^{\perp}, \quad t=1, \ldots, \text { ind } \mathcal{F} .
$$

Таким образом, метод орбит коприсоединенного представления позволяет детально изучить структуру $\mathcal{F}$-алгебры инвариантных операторов. В частности, на основе приведенных построений можно сравнительно легко предъявить явный вид данных операторов.

На симплектических листах (8) дуального пространства $\mathcal{F}^{*}$ перейдем к каноническим координатам Дарбу $(u, v)$. Как и в случае К-орбит, возможно определить каноническое вложение $a_{\mu}: O_{\sigma} \rightarrow \mathcal{F}^{*}$, которое задается функциями $a_{\mu}=a_{\mu}(u, v, \sigma)$, удовлетворяющими системе уравнений $\left\{a_{\mu}, a_{\nu}\right\}=\Omega_{\mu \nu}^{\mathrm{cl}}(a), \mu, \nu=1, \ldots, \operatorname{dim} \mathcal{F}$.

Приступим к процедуре квантования симплектических листов. Для этого заменим функции $a_{\mu}(u, v, \sigma)$ на дифференциальные операторы $\hat{a}_{\mu}\left(v, i \partial_{v}, \tilde{\sigma}\right)$, действующие в пространстве функций, определенных на лагранжевом подмногообразии симплектического листа (функций от переменных $v \in V$ ), и наложим коммутационные соотношения $-i\left[\hat{a}_{\mu}, \hat{a}_{\nu}\right]=\Omega_{\mu \nu}(\hat{a})$. В операторы $\hat{a}_{\mu}\left(v, i \partial_{v}, \tilde{\sigma}\right)$ введен "квантовый сдвиг" $\sigma \rightarrow \tilde{\sigma}$, возникающий при квантовании К-орбит $\left(s_{M}\right)$-типа, соответствующих однородному пространству. Имеют место следующие тождества:

$$
Z_{t}(\hat{a})=\tilde{\sigma}_{t}, \quad D_{t}(\tilde{\sigma})=\kappa_{t}^{\left(s_{M}\right)}(\sigma)
$$

Реализация $\mathcal{F}$-алгебры операторами $\hat{a}$, так что выполняются условия $(9)$, называется $\lambda$-представлением $\mathcal{F}$-алгебры [19].

Рассмотрим процедуру построения гармонического анализа для функций на однородном пространстве $M$, принадлежащих гильбертову пространству $L_{2}(M, d \mu(x))$, где $d \mu(x)$ - риманова мера, построенная по инвариантной метрике. 
Над областями тривиализации $U \in P$ в расслоенном пространстве $G$ введем координаты $g^{A}=\left(x^{a}, h^{\alpha}\right)$ прямого произведения $U \times H(a=1, \ldots, \operatorname{dim} M$, $\alpha=\operatorname{dim} M+1, \ldots, \operatorname{dim} G)$. При этом координаты произвольной точки $g \in G$ можно представить в виде $g=h s(x)$, где $s: M \rightarrow G$ - локальное гладкое сечение расслоения $G$. Любой гладкой функции $\varphi(g)$ на группе Ли $G$, постоянной на слоях $H$ главного расслоения $(G, \pi, M, H)$, однозначно соответствует функция $\left(\pi^{*} \varphi\right)(x)=\varphi(s(x))$ на однородном пространстве $M$ (проекция функции $\varphi$ на $M$ ). Другими словами, имеет место изоморфизм $C^{\infty}(M) \simeq \mathcal{F}_{G}$, где функциональное пространство $\mathcal{F}_{G}$, ввиду связности группы Ли $H$, определяется равенством

$$
\mathcal{F}_{G} \equiv\left\{\varphi \in C^{\infty}(G) \mid \eta_{X} \varphi(g)=0, X \in \mathfrak{h}, g \in G\right\}
$$

Так как $F^{\left(s_{M}\right)}(\xi)=0$, то пространство $L_{2}(M, d \mu(x))$ изоморфно пространству

$$
L_{\left(s_{M}\right)}(M)=\left\{\varphi \in L_{\left(s_{M}\right)} \mid \eta_{X} \varphi=0, X \in \mathfrak{h}\right\} .
$$

Таким образом, гармонический анализ на однородном пространстве $M$ сводится к гармоническому анализу в пространстве $L_{\left(s_{M}\right)}$.

Инвариантному пространству $L_{\left(s_{M}\right)}$ соответствует инвариантное подпространство $M_{\left(s_{M}\right)}$, состоящее из K-орбит $\mathcal{O}_{\lambda}^{\left(s_{M}\right)}$, представители которых лежат в $\mathfrak{h}^{\perp}$. Проведем квантование $\mathrm{K}$-орбит и построим $\lambda$-представление алгебры $\mathfrak{g}$, соответствующее K-орбите $\mathcal{O}_{\lambda}^{\left(s_{M}\right)}$. Так как $\lambda \in \mathfrak{h}^{\perp}$, то на параметры $\lambda$ налагается условие $\tilde{K}_{\mu}^{\left(s_{M}\right)}(\lambda)=0, \mu=1, \ldots, r_{\left(s_{M}\right)} \cdot \lambda$-Представление, удовлетворяющее данному требованию, в работе [19] названо соответствующим однородному пространству $M$.

Поднимем $\lambda$-представление алгебры $\mathfrak{g}\left(s_{M}\right)$-типа, соответствующее однородному пространству $M$, до представления группы Ли $G$. Набор функций $D_{q \bar{q}^{\prime}}^{\lambda}\left(g^{-1}\right)$ в силу полноты и ортогональности образует базис функционального пространства $L_{\left(s_{M}\right)}$.

Определим параметрическое семейство функций $D_{q v}^{\lambda}(x)$, задающих базис в функциональном пространстве $L_{2}(M, d \mu(x)) \simeq L_{\left(s_{M}\right)} \cap \mathcal{F}_{G}$, в виде разложения по функциям $D_{q \bar{q}^{\prime}}^{\lambda}\left(g^{-1}\right)$ :

$$
D_{q v}^{\lambda}(x)=\int c_{\lambda}\left(q^{\prime}, v\right) D_{q q^{\prime}}^{\lambda}\left(g^{-1}\right) d \mu\left(q^{\prime}\right), \quad g=(x, h) .
$$

На коэффициенты разложения наложим условия

$$
\begin{gathered}
l_{\alpha}\left(q^{\prime}, \lambda\right) c_{\lambda}\left(q^{\prime}, v\right)=0, \\
{\left[L_{\mu}\left(-i l\left(q^{\prime}, \lambda\right)\right)-\hat{a}_{\mu}(v, \lambda)\right] c_{\lambda}\left(q^{\prime}, v\right)=0 .}
\end{gathered}
$$

В силу условия (11) правая часть $(10)$ принадлежит классу функций $\mathcal{F}_{G}$. При выполнении (12) функции $D_{q v}^{\lambda}(x)$ образуют базис $\lambda$-представления $\mathcal{F}$-алгебры инвариантных функций.

В силу соотношений $(2)$ функции $D_{q v}^{\lambda}(x)$ удовлетворяют системе уравнений

$$
\left[X_{A}(x)+l_{A}(q, \lambda)\right] D_{q v}^{\lambda}(x)=0, \quad\left[L_{\mu}(x)-\hat{a}_{\mu}(v, \lambda)\right] D_{q v}^{\lambda}(x)=0,
$$

где $A=1, \ldots, \operatorname{dim} \mathfrak{g}, \mu=1, \ldots, \operatorname{dim} \mathcal{F}$. Определим семейство обобщенных функций $\tilde{D}_{q v}^{\lambda}(x)$ как решение системы

$$
\left[X_{A}^{\dagger}(x)+l_{A}^{\dagger}(q, \lambda)\right] \tilde{D}_{q v}^{\lambda}(x)=0, \quad\left[L_{\mu}^{\dagger}(x)-\hat{a}_{\mu}^{\dagger}(v, \lambda)\right] \tilde{D}_{q v}^{\lambda}(x)=0 .
$$


Так как обобщенные функции $D_{q \overline{q^{\prime}}}^{\lambda}\left(g^{-1}\right)$ обладают свойствами полноты и ортогональности в функциональном пространстве $L_{s_{M}}$, то семейство обобщенных функций $D_{q v}^{\lambda}(x)$ и $\tilde{D}_{q v}^{\lambda}(x)$, где $\lambda$ - невырожденный ковектор $\left(s_{M}\right)$-типа, образует полный и ортогональный набор в функциональном пространстве $L_{2}(M, d \mu(x))$ :

$$
\begin{gathered}
\int_{M} \overline{\tilde{D}_{\tilde{q} \tilde{v}}^{\tilde{\lambda}}}(x) D_{q v}^{\lambda}(x) d \mu(x)=\delta(q, \overline{\tilde{q}}) \delta(\tilde{v}, v) \delta(\tilde{\lambda}, \lambda), \\
\int_{Q \times V \times J} \overline{\tilde{D}_{q v}^{\lambda}(\tilde{x})} D_{q v}^{\lambda}(x) d \mu(q) d \mu(v) d \mu(\lambda)=\delta(\tilde{x}, x) .
\end{gathered}
$$

Соотношения $(13),(14)$ позволяют ввести аналоги прямого и обратного преобразований Фурье на однородном пространстве $M$. Пусть $\Phi(M) \subset L_{2}(M, d \mu(x)) \subset \Phi^{\prime}(M)-$ триплет Гельфанда. Пространство $\Phi(M)$ является линейным пространством функций $\varphi(x) \in L_{2}(M, d \mu(x))$, для которых определено обобщенное преобразование Фурье:

$$
\psi_{\lambda}(q, v)=\int_{M} \varphi(x) \overline{\tilde{D}_{q v}^{\lambda}(x)} d \mu(x) .
$$

Функции $\psi_{\lambda}(q, v)$ образуют линейное пространство $\widehat{\Phi}(M)$, двойственное к $\Phi(M)$. Обратное преобразование имеет вид

$$
\varphi(x)=\int_{Q \times V \times J} \psi_{\lambda}(q, v) D_{q v}^{\lambda}(x) d \mu(q) d \mu(v) d \mu(\lambda) .
$$

Преобразования (15), (16) дают вид взаимно однозначного непрерывного отображения пространств $\Phi(M)$ и $\widehat{\Phi}(M)$. По аналогии с гармоническим анализом на группах Ли считаем, что дифференциальные операторы $X_{A}, L_{\mu}$, действующие в пространстве $\Phi(M)$, в двойственном пространстве $\widehat{\Phi}(M)$ также являются дифференциальными:

$$
X_{A}(x) \Longleftrightarrow-\overline{l_{A}^{\dagger}(q, \lambda)}, \quad L_{\mu}(x) \Longleftrightarrow \overline{a_{\mu}^{\dagger}(q, \lambda)} .
$$

Заметим, что операторы Казимира $K_{t}^{\left(s_{M}\right)}(i X)=D_{t}(Z(L))$ в пространстве $\widehat{\Phi}(M)$ являются операторами умножения на $\kappa_{t}^{\left(s_{M}\right)}(\lambda)$.

\section{4. НЕКОММУТАТИВНАЯ РЕДУКЦИЯ УРАВНЕНИЯ КЛЕЙНА-ГОРДОНА}

Введем на однородном пространстве $M$ инвариантную метрику. Пусть $\mathbf{G}$ - невырожденная квадратичная форма на подпространстве $\mathfrak{m} \subset \mathfrak{g}$, удовлетворяющая условию $\mathrm{Ad}_{H}$-инвариантности:

$$
\mathbf{G}(\overline{[X, Y]}, \bar{Z})+\mathbf{G}(\bar{Y}, \overline{[X, Z]})=0, \quad X \in \mathfrak{h}, \quad Y, Z \in \mathfrak{g},
$$

где черта сверху означает проекцию элемента алгебры Ли $\mathfrak{g}$ на подпространство $\mathfrak{m}$. Квадратичная форма $\mathbf{G}$ задает $\mathrm{Ad}_{H}$-инвариантное скалярное произведение на касательном пространстве $T_{x_{0}} M \simeq \mathfrak{m}$. Требование $\operatorname{Ad}_{H}$-инвариантности (17) позволяет при помощи действия группы $G$ корректно доопределить данное скалярное произведение на все однородное пространство $M$ :

$$
g_{M}(\tau, \sigma)(x)=\mathbf{G}\left(\left(R_{g^{-1}}\right)_{*} \tau,\left(R_{g^{-1}}\right)_{*} \sigma\right), \quad \tau, \sigma \in T_{x} M, \quad x=\pi(g), \quad g \in G .
$$


Скалярное произведение (18) определяет инвариантную метрику $g_{M}$ на однородном пространстве $M[26]$.

Приведем выражение для метрического тензора инвариантной метрики $g_{M}$ в локальных координатах на пространстве $M$ :

$$
\left(g_{M}\right)_{i j}(x)=G_{a b} \sigma_{i}^{a}(x) \sigma_{j}^{b}(x), \quad G_{a b} \equiv \mathbf{G}\left(e_{a}, e_{b}\right) .
$$

Здесь $\left\{e_{a}\right\}$ - некоторый фиксированный базис в пространстве $\mathfrak{m}, \sigma^{b}(x)$ - базисные правоинвариантные 1-формы: $\sigma^{b}(y) \equiv\left(R_{g}\right)^{*} e^{b},\left\{e^{b}\right\}-$ базис в $\mathfrak{m}^{*}:\left\langle e_{a}, e^{b}\right\rangle=\delta_{a}^{b}$. Для контравариантных компонент метрического тензора соответственно имеем

$$
\left(g_{M}\right)^{i j}=G^{a b} \eta_{A}^{i}(x) \eta_{B}^{j}(x), \quad G^{a b}=\left(G_{a b}\right)^{-1} .
$$

По повторяющимся индексам, находящимся вверху и внизу, подразумевается суммирование.

Будем считать, что на однородном пространстве $M$ задана инвариантная метрика $g_{M}$ лоренцевой сигнатуры. Мы рассматриваем модель статического пространства-времени, когда на $M$ существует глобальный времениподобный вектор Киллинга $X_{0}=\partial_{x_{0}}$, ортогональный гиперповерхностям постоянного времени $\Sigma: x_{0}=$ const. Тогда $x=(\bar{x}, t)$, где $\bar{x}=\left(x^{1}, \ldots, x^{\operatorname{dim} M-1}\right)$ - локальные координаты на гиперповерхности $\Sigma, t=x_{0}$ - переменная, играющая роль времени. Считаем, что метрический тензор зависит только от локальных координат на гиперповерхности $\Sigma: g_{M}=g_{M}(\bar{x})$.

Уравнение Клейна-Гордона является уравнением Эйлера-Лагранжа относительно действия $S=\int \mathcal{L}^{0} d \mu(x)$ скалярного поля $\varphi(x)$ на однородном пространстве $M$ с лагранжианом

$$
\mathcal{L}^{0}\{\bar{\varphi}, \varphi\}=\frac{\sqrt{-\mathrm{G}}}{2}\left(G^{a b} \eta_{a} \overline{\varphi(x)} \eta_{b} \varphi(x)-\left[m^{2}+\zeta R\right]|\varphi(x)|^{2}\right)
$$

где $R$ - скалярная кривизна однородного пространства $M, \zeta=(\operatorname{dim} M-2) / 4(\operatorname{dim} M-$ 1) - конформный множитель, обеспечивающий связь гравитационного поля с кривизной, $\mathrm{G}=\operatorname{det}\left(G_{a b}\right)$. Тогда уравнение Клейна-Гордона на $M$ имеет вид

$$
\left(\Delta_{M}+m^{2}+\zeta R\right) \varphi(x)=0
$$

где $\Delta_{M}$ - оператор Лапласа инвариантной метрики на однородном пространстве $M$ :

$$
\Delta_{M}=G^{a b}\left(\eta_{a} \eta_{b}-2 c_{a} \eta_{b}\right), \quad c_{a} \equiv \frac{1}{2} \operatorname{Sp}\left(\left.\operatorname{ad}_{a}\right|_{\mathfrak{m}}\right)
$$

Нашей целью является построение базиса решений $\varphi_{\sigma}(x)$ уравнения $(20)$, нумеруемого коллективным индексом $\sigma$ и удовлетворяющего условию нормировки скалярного поля:

$$
-i \int \overline{\varphi_{\sigma}(x)} \overleftrightarrow{X_{0}} \varphi_{\sigma^{\prime}}(x) \sqrt{-g_{M}(\bar{x})} d \bar{x}=\delta\left(\sigma, \sigma^{\prime}\right), \quad g_{M}(\bar{x})=\operatorname{det}\left(\left(g_{M}\right)_{i j}(\bar{x})\right)
$$

Положительно- и отрицательно-частотные решения $\varphi_{\sigma}^{ \pm}(x)$ определим как собственные функции времениподобного вектора Киллинга $X_{0}$ :

$$
X_{0}(x) \varphi_{\sigma}^{( \pm)}(x)=\mp i \omega \varphi_{\sigma}^{( \pm)}(x), \quad \omega \in \sigma .
$$


Заметим, что уравнение (20) можно рассматривать как волновое уравнение на группе Ли $G$ преобразований в классе функций $\mathcal{F}_{G}$. Поэтому редукцию уравнения (20) проведем аналогично редукции квантовых уравнений на группе Ли. Из (23) следует

$$
D_{q v}^{\lambda}(\bar{x}, t)=e^{-i \omega t} D_{q v}^{\lambda^{\prime}}(\bar{x}, 0), \quad l_{0}=i \omega, \quad \hat{a}_{0}=\omega,
$$

где параметр $\omega$ входит в набор параметров $\lambda=\left(\omega, \lambda^{\prime}\right)$, нумерующих орбиты.

Введем операторы $\eta_{X}^{\prime} \equiv \eta_{X}-c_{X}$, которые являются тривиалъным продолжениeм векторных полей $\eta_{X}:\left[\eta_{X}^{\prime}, \eta_{Y}^{\prime}\right]=\eta_{[X, Y]}^{\prime}, X, Y \in \mathfrak{g}$. Векторные поля $\eta_{X}^{\prime}$ эрмитовы относительно римановой меры, и оператор (21) можно представить как симметрическую форму от $\eta_{X}^{\prime}$ :

$$
\Delta_{M}=H^{\prime}\left(\eta^{\prime}\right)=G^{a b} \eta_{a}^{\prime} \eta_{b}^{\prime}-G^{a b} c_{a} c_{b}
$$

Так как генераторы группы Ли преобразований $X$ являются векторами Киллинга инвариантной метрики $g_{M}(x)$, то оператор Лапласа $\Delta_{M}$ на однородном пространстве $M$ коммутирует с $X$ и, следовательно, представим как дифференциальный оператор, выражающийся полиномиально через инвариантные операторы $L_{\mu}\left(-i \eta^{\prime}\right) \equiv L_{\mu}(-i \eta+i c): \Delta_{M}=H(L(x))$. В отличие от $L_{\mu}(-i \eta)$, операторы $L_{\mu}\left(-i \eta^{\prime}\right)$ эрмитовы относительно римановой меры.

Уравнение (12) в нашем случае имеет вид

$$
\left[L_{\mu}\left(-i l\left(q^{\prime}, \lambda\right)+i c\right)-\hat{a}_{\mu}(v, \lambda)\right] c_{\lambda}\left(q^{\prime}, v\right)=0 .
$$

Выберем меру $d \mu(v)$, относительно которой операторы $\hat{a}_{\mu}(v, \lambda)$ эрмитовы. Тогда $\tilde{D}_{q v}^{\lambda}(x)=\Delta^{-1}(q) D_{q v}^{\lambda}(x)$.

При помощи преобразования (16) редуцируем уравнение (20) к уравнению на симплектическом листе $\mathcal{F}$-алгебры инвариантных функций. Переменные $q$ будут входить в редуцированное уравнение как параметры, поэтому набор

$$
\varphi_{\sigma}(\bar{x}, t)=\frac{1}{\sqrt{2 \omega \Delta(q) \sqrt{-G}}} e^{-i \omega t} \int \psi_{\omega}\left(v, \lambda^{\prime}\right) D_{q v}^{\lambda^{\prime}}(\bar{x}, 0) d \mu(v), \quad \sigma=(q, \lambda),
$$

образует базис решений уравнения (20). Функции $\psi_{\omega}\left(v, \lambda^{\prime}\right)$ находятся из редуцированного уравнения с $d(M)$ независимыми переменными на симплектическом листе:

$$
\left(H(\overline{\hat{a}(v, \lambda)})+m^{2}+\zeta R\right) \psi_{\omega}\left(v, \lambda^{\prime}\right)=0 .
$$

Уравнение (25) является обыкновенным дифференциальным уравнением при выполнении условия $d(M)<2$.

Так как оператор уравнения (25) эрмитов, собственные функции $\psi_{\omega}(v, \lambda)$ удовлетворяют условию ортогональности

$$
\int_{Q} \overline{\psi_{\omega}\left(q^{\prime}, \lambda^{\prime}\right)} \psi_{\omega^{\prime}}\left(q^{\prime}, \lambda^{\prime}\right) d \mu\left(q^{\prime}\right)=\delta\left(\omega, \omega^{\prime}\right) .
$$

Выполнение данного соотношения влечет за собой выполнение условия нормировки (22) для полного набора решений (24). Заметим, что требование (26) является условием нормировки функций $\psi_{\omega}\left(v, \lambda^{\prime}\right)$ и определением меры по $\omega$. 
Рассмотрим обобщенную дзета-функцию оператора $\widehat{F}=\Delta_{M}+\zeta R+m^{2}$ уравнения Клейна-Гордона

$$
\zeta(s)=\int \theta_{\sigma}^{-s} d \mu(\sigma)
$$

где $\theta_{\sigma}$ - собственные значения оператора $\widehat{F}$, нумеруемые коллективным индексом $\sigma$. Обобщенная дзета-функция $\zeta(s)$ допускает аналитическое продолжение в комплексную плоскость, регулярное в точке $s=0$, и может быть определена как интеграл по $M$ от локальной дзета-функции:

$$
\zeta(\bar{x}, t ; s)=\int \theta_{\sigma}^{-s} \overline{\phi_{\sigma}(\bar{x}, t)} \phi_{\sigma}(\bar{x}, t) d \mu(\sigma), \quad \zeta(s)=\sqrt{-G} \int \zeta(\bar{x}, t ; s) d \mu(x),
$$

где $\phi_{\sigma}(\bar{x}, t)$ - полный и ортогональный набор собственных функций оператора $\widehat{F}$, удовлетворяющий условию нормировки

$$
\sqrt{-G} \int \overline{\phi_{\sigma}(\bar{x}, t)} \phi_{\sigma^{\prime}}(\bar{x}, t) d \mu(x)=\delta\left(\sigma, \sigma^{\prime}\right) .
$$

Как и при редукции уравнения Клейна-Гордона, собственные функции $\phi_{\sigma}(\bar{x}, t)$ будем искать в виде

$$
\phi_{\sigma}(\bar{x}, t)=(\Delta(q) \sqrt{-G})^{-1 / 2} \int \tilde{\psi}_{\nu}(v, \lambda) D_{q v}^{\lambda}(\bar{x}, t) d \mu(v), \quad \sigma=(q, \lambda, \nu) .
$$

Тогда задача на собственные значения $\widehat{F} \phi_{\sigma}(\bar{x}, t)=\theta_{\sigma} \phi_{\sigma}(\bar{x}, t)$ сводится к уравнению на симплектическом листе

$$
H(\overline{\hat{a}(v, \lambda)}) \tilde{\psi}_{\nu}(v, \lambda)=\left(\theta_{\nu \lambda}-\zeta R-m^{2}\right) \tilde{\psi}_{\nu}(v, \lambda) .
$$

Спектр оператора $H(\overline{\hat{a}(v, \lambda)})$ определяется дополнительным квантовым числом $\nu$ и не зависит от $q$. На функции $\tilde{\psi}_{\nu}(v, \lambda)$ налагается условие

$$
\int \overline{\tilde{\psi}_{\nu}(v, \lambda)} \tilde{\psi}_{\nu^{\prime}}(v, \lambda) d \mu(v)=\delta\left(\nu, \nu^{\prime}\right)
$$

Для локальной дзета-функции получаем выражение

$$
\begin{gathered}
\zeta_{\text {loc }}(s)=\frac{1}{\sqrt{-G}} \int \theta_{\nu \lambda}^{-s}\left(\int \tilde{\psi}_{\nu}(v, \lambda) \tilde{\psi}_{\nu}(\tilde{v}, \lambda) \chi^{\lambda}(v, \tilde{v}) d \mu(v) d \mu(\tilde{v})\right) d \mu(\lambda) d \mu(\nu), \\
\chi^{\lambda}(v, \tilde{v})=\int \overline{D_{q v}^{\lambda}(x)} D_{q \tilde{v}}^{\lambda}(x) d \mu_{0}(q) .
\end{gathered}
$$

Покажем, что интеграл (29) не зависит от локальных координат. Подставляя разложение (10), получим

$$
\chi^{\lambda}(v, \tilde{v})=\int \overline{c_{\lambda}\left(q^{\prime}, v\right)} c_{\lambda}\left(\tilde{q}^{\prime}, \tilde{v}\right) I_{q^{\prime} \tilde{q}^{\prime}}^{\lambda} d \mu\left(q^{\prime}\right) d \mu\left(\tilde{q}^{\prime}\right), \quad I_{q^{\prime} \tilde{q}^{\prime}}^{\lambda}=\int \overline{D_{q \overline{q^{\prime}}}^{\lambda}\left(g^{-1}\right)} D_{q \tilde{q}^{\prime}}^{\lambda}\left(g^{-1}\right) d \mu_{0}(q) .
$$

Из системы уравнений $(2)$ следует $\overline{D_{q \bar{q}^{\prime}}^{\lambda}\left(g^{-1}\right)}=\left(\Delta(q) / \Delta\left(q^{\prime}\right)\right) D_{q^{\prime} \bar{q}}^{\lambda}(g)$, и интеграл $I_{q^{\prime} \tilde{q}^{\prime}}^{\lambda}$ равен

$$
I_{q^{\prime} \tilde{q}^{\prime}}^{\lambda}=\frac{1}{\Delta\left(q^{\prime}\right)} \int D_{q^{\prime} \bar{q}}^{\lambda}(g) D_{q \overline{\tilde{q}^{\prime}}}^{\lambda}\left(g^{-1}\right) d \mu(q)=\frac{1}{\Delta\left(q^{\prime}\right)} D_{q^{\prime} \bar{q}^{\prime}}^{\lambda}(0)=\delta_{0}\left(q^{\prime}, \overline{\tilde{q}^{\prime}}\right) .
$$


Тогда для $\chi^{\lambda}(v, \tilde{v})$ имеем следующее выражение, не содержащее локальных координат:

$$
\chi^{\lambda}(v, \tilde{v})=\int \overline{c_{\lambda}\left(q^{\prime}, v\right)} c_{\lambda}\left(q^{\prime}, \tilde{v}\right) d \mu_{0}\left(q^{\prime}\right) .
$$

Если однородное пространство является группой Ли, то $c\left(q^{\prime}, v\right)=\delta\left(q^{\prime}, v\right)$ и выражение (30) дает дельта-функцию Дирака [18]. Отметим, что обобщенная дзета-функция выражается через набор решений редуцированного уравнения и определяется через алгебраические характеристики однородного пространства.

\section{5. ВАКУУМНЫЕ СРЕДНИЕ ТЭИ СКАЛЯРНОГО ПОЛЯ НА ОДНОРОДНОМ ПРОСТРАНСТВЕ}

Метрический ТЭИ скалярного поля на однородном пространстве $M$ определяется вариацией действия скалярного поля с лагранжианом (19) по метрике [1]

$$
\delta S=-\frac{1}{2} \int \sqrt{-g_{M}(x)}\left\langle T, \delta g_{M}(x)\right\rangle d \mu(x) d t,
$$

где $\langle\cdot, \cdot\rangle$ - свертка ТЭИ $T$ с вариацией метрического тензора $g_{M}(x)$. Выражение для ТЭИ скалярного поля в локальных координатах хорошо известно и в наших обозначениях имеет вид [1]

$$
\begin{aligned}
T_{i j}\{\varphi, \bar{\varphi}\}= & \left.(1-2 \zeta) \overline{\varphi_{(, i}} \varphi_{, j}\right)+\left(2 \zeta-\frac{1}{2}\right) g_{i j} g^{k l} \overline{\varphi, k} \varphi, l-\zeta\left[\left(\nabla_{i} \nabla_{j} \bar{\varphi}\right) \varphi+\bar{\varphi}\left(\nabla_{i} \nabla_{j} \varphi\right)\right]- \\
& -\left[\zeta R_{i j}+\left(2 \zeta-\frac{1}{2}\right) g_{i j}\left(m^{2}+\zeta R\right)\right]|\varphi|^{2},
\end{aligned}
$$

где $(a, b) \equiv(a b+b a) / 2, \Delta_{i}-$ ковариантная производная связности Леви-Чивиты на однородном пространстве $M$ с инвариантной метрикой, $R_{i j}$ - тензор Риччи.

Домножим выражение (31) на $\eta_{X}^{i}(x) \eta_{Y}^{j}(x)$ и, воспользовавшись свойствами инвариантной метрики на однородном пространстве, получим

$$
\begin{aligned}
T^{\prime}(X, Y)\{\bar{\varphi}, \varphi\}= & T\left(\eta_{X}, \eta_{Y}\right)\{\bar{\varphi}, \varphi\}=T_{i j}\{\bar{\varphi}, \varphi\} \eta_{X}^{i}(x) \eta_{Y}^{j}(x)= \\
= & \left(2 \zeta-\frac{1}{2}\right) \overline{\eta_{(X} \varphi} \eta_{Y)} \varphi+\left(2 \zeta-\frac{1}{2}\right) \mathbf{G}(X, Y) G^{A B} \overline{\eta_{A} \varphi} \eta_{B} \varphi- \\
& -\left[\zeta \operatorname{Ric}(X, Y)+\left(2 \zeta-\frac{1}{2}\right) \mathbf{G}(X, Y)\left(m^{2}+\zeta R\right)\right] \bar{\varphi} \varphi- \\
& -\zeta \eta_{X}^{i} \eta_{Y}^{j}\left[\left(\nabla_{i} \nabla_{j} \bar{\varphi}\right) \varphi+\bar{\varphi}\left(\nabla_{i} \nabla_{j} \varphi\right)\right], \quad X, Y \in \mathfrak{m},
\end{aligned}
$$

где мы ввели обозначение $\boldsymbol{\operatorname { R i c }}(X, Y)=R_{i j} \eta_{X}^{i}(x) \eta_{Y}^{j}(x)$. Используя выражение для символов Кристоффеля связности Леви-Чивиты на однородном пространстве $M$ в локальных координатах, путем простых, но громоздких выкладок можно получить

$$
\eta_{X}^{i} \eta_{Y}^{j} \nabla_{i} \nabla_{j} \varphi=\eta_{X} \eta_{Y}-\eta_{\Gamma(X, Y)}
$$

где билинейное отображение $\boldsymbol{\Gamma}: \mathfrak{m} \times \mathfrak{m} \rightarrow \mathfrak{m}$ задает связность Леви-Чивиты и однозначно определяется из системы уравнений

$$
2 \mathbf{G}(\overline{\boldsymbol{\Gamma}(X, Y)}, Z)=\mathbf{G}(\overline{[X, Y]}, Z)+\mathbf{G}(\overline{[Y, Z]}, X)-\mathbf{G}(\overline{[Z, X]}, Y), \quad X, Y, Z \in \mathfrak{m}
$$


Заметим, что величина $\boldsymbol{R i c}(X, Y)$ не зависит от локальных координат и может быть определена как след линейного оператора $Z \rightarrow \mathbf{R}(Z, Y) X, X, Y, Z \in \mathfrak{m}$, где

$$
\mathbf{R}(X, Y) Z=\boldsymbol{\Gamma}(Y, \boldsymbol{\Gamma}(X, Z))-\boldsymbol{\Gamma}(X, \boldsymbol{\Gamma}(Y, Z))+\boldsymbol{\Gamma}(\overline{[X, Y]}, Z), \quad X, Y, Z \in \mathfrak{m} .
$$

В итоге для $T^{\prime}(X, Y)$ получим выражение

$$
\begin{aligned}
T^{\prime}(X, Y)\{\bar{\varphi}, \varphi\}=(1-2 \zeta) \overline{\eta_{(X} \varphi} \eta_{Y)} \varphi+ \\
\quad+(4 \zeta-1) \mathbf{G}(X, Y) \frac{1}{\sqrt{-G}} \mathcal{L}^{0}\{\bar{\varphi}, \varphi\}-\zeta \mathbf{R i c}(X, Y)|\varphi|^{2}- \\
\quad-\zeta\left[\overline{\left(\eta_{(X} \eta_{Y)}-\eta_{Z(X, Y)}\right) \varphi \varphi}+\bar{\varphi}\left(\eta_{(X} \eta_{Y)}-\eta_{Z(X, Y)}\right) \varphi\right], \quad X, Y \in \mathfrak{m},
\end{aligned}
$$

где $Z(X, Y)=(\boldsymbol{\Gamma}(X, Y)+\boldsymbol{\Gamma}(Y, X)) / 2$. Компоненты (34) будем называть квазитетрадными компонентами ТЭИ скалярного поля (31). Заметим, что выражение (34) не зависит от локальных координат на однородном пространстве и в силу соотношений

$$
\sigma_{b}^{a}(x) \eta_{a}^{c}(x)=\delta_{b}^{a}, \quad \sigma_{c}^{a}(x) \eta_{b}^{c}(x)=\delta_{b}^{a}, \quad a, b, c=1, \ldots, \operatorname{dim} M
$$

всегда существует возможность перейти к обычным компонентам ТЭИ:

$$
T_{i j}=T^{\prime}\left(e_{a}, e_{b}\right) \sigma_{i}^{a}(x) \sigma_{j}^{b}(x) .
$$

Соотношения (31)-(35) представляют собой аналог тетрадного формализма для тензорных полей на однородном пространстве и очевидным образом обобщаются на случай тензорных полей произвольного ранга.

Проведем процедуру квантования скалярного поля с лагранжианом (19) на однородном пространстве $M$. Разложим оператор поля $\hat{\phi}(\bar{x}, t)$ по базису решений волнового уравнения (20):

$$
\hat{\phi}(\bar{x}, t)=\int d \mu(\sigma)\left[\varphi(\bar{x}, t) \hat{a}_{\sigma}+\overline{\varphi(\bar{x}, t)} \hat{a}_{\sigma}^{\dagger}\right],
$$

где $d \mu(\sigma)$ - мера по всем квантовым числам, $\hat{a}_{\sigma}^{\dagger}, \hat{a}_{\sigma}-$ операторы рождения и уничтожения соответственно. Ковариантное квантование осуществляется наложением коммутационных соотношений $\left[\hat{a}_{\sigma}, \hat{a}_{\sigma^{\prime}}^{\dagger}\right]=\delta\left(\sigma, \sigma^{\prime}\right)$. Вакуумное состояние, соответствующее данной процедуре квантования, определяется условием $\hat{a}_{\sigma}|0\rangle=0$. Тогда вакуумные средние ТЭИ определяются интегралом по всем квантовым числам:

$$
\left\langle\widehat{T}^{\prime}(X, Y)\right\rangle=\int T^{\prime}(X, Y)\left\{\varphi_{\sigma}, \bar{\varphi}_{\sigma}\right\} d \mu(\sigma), \quad X, Y \in \mathfrak{m} .
$$

Для вычисления интеграла (36) найдем выражение для вакуумных средних вида

$$
\Phi(X, Y)=\left\langle\eta_{X} \hat{\varphi}, \eta_{Y} \hat{\varphi}\right\rangle=\int \overline{\left(\eta_{X} \varphi_{\sigma}\right)} \eta_{Y} \varphi_{\sigma} d \mu(q) d \mu(\lambda), \quad X, Y \in \mathfrak{m}
$$

Подставляя (24) и учитывая равенство

$$
\eta_{X} D_{q v}^{\lambda}(x, t)=\int\left(l_{X}\left(q^{\prime}, \lambda\right) c_{\lambda}\left(q^{\prime}, v\right)\right) D_{q \overline{q^{\prime}}}^{\lambda}\left(g^{-1}\right) d \mu\left(q^{\prime}\right)
$$


для средних $\Phi(X, Y)$ имеем цепочку равенств

$$
\begin{aligned}
\Phi(X, Y)= & \frac{1}{2 \sqrt{-G}} \int \frac{1}{\omega} \overline{\left(\psi_{\omega}\left(v, \lambda^{\prime}\right) l_{X}\left(q^{\prime}, \lambda\right) c_{\lambda}\left(q^{\prime}, v\right)\right)}\left(\psi_{\omega}\left(\tilde{v}, \lambda^{\prime}\right) l_{X}\left(\tilde{q}^{\prime}, \lambda\right) c_{\lambda}\left(\tilde{q}^{\prime}, \tilde{v}\right)\right) \times \\
& \times I_{q^{\prime} \tilde{q}^{\prime}}^{\lambda} d \mu\left(q^{\prime}\right) d \mu\left(\tilde{q}^{\prime}\right) d \mu(v) d \mu(\tilde{v}) d \mu(\lambda)= \\
= & \frac{1}{2 \sqrt{-G}} \int \frac{1}{\omega} \overline{\left(\psi_{\omega}\left(v, \lambda^{\prime}\right) l_{X}\left(q^{\prime}, \lambda\right) c_{\lambda}\left(q^{\prime}, v\right)\right)}\left(\psi_{\omega}\left(\tilde{v}, \lambda^{\prime}\right) l_{Y}\left(q^{\prime}, \lambda\right) c_{\lambda}\left(q^{\prime}, \tilde{v}\right)\right) \times \\
& \times d \mu_{0}\left(q^{\prime}\right) d \mu(v) d \mu(\tilde{v}) d \mu(\lambda) .
\end{aligned}
$$

Действуя аналогично, для вакуумных средних квадрата оператора поля $\left\langle\hat{\varphi}^{2}\right\rangle \equiv$ $\langle\hat{\varphi}, \hat{\varphi}\rangle$ получим

$$
\left\langle\hat{\varphi}^{2}\right\rangle=\frac{1}{2 \sqrt{-G}} \int \frac{1}{\omega} \overline{\psi_{\omega}\left(v, \lambda^{\prime}\right)} \psi_{\omega}\left(\tilde{v}, \lambda^{\prime}\right) \chi^{\lambda}(v, \tilde{v}) d \mu(v) d \mu(\tilde{v}) d \mu(\lambda) .
$$

Заметим, что в (37) операторы $\lambda$-представления косоэрмитовы относительно $d \mu_{0}\left(q^{\prime}\right)$, откуда следует, что $\left\langle\eta_{X} \eta_{Y} \hat{\varphi}, \hat{\varphi}\right\rangle=-\Phi(X, Y),\left\langle\eta_{X} \hat{\varphi}, \hat{\varphi}\right\rangle=-\left\langle\hat{\varphi}, \eta_{X} \hat{\varphi}\right\rangle$. Тогда мы можем выразить вакуумные средние ТЭИ через $\Phi(X, Y)$ и $\left\langle\hat{\varphi}^{2}\right\rangle$ :

$$
\begin{aligned}
\left\langle\widehat{T}^{\prime}(X, Y)\right\rangle= & \frac{1}{2}(\Phi(X, Y)+\Phi(Y, X))-\zeta \mathbf{R i c}(X, Y)\left\langle\hat{\varphi}^{2}\right\rangle+ \\
& +\left(2 \zeta-\frac{1}{2}\right) \mathbf{G}(X, Y)\left[G^{a b} \Phi\left(e_{a}, e_{b}\right)-\left(m^{2}+\zeta R\right)\left\langle\hat{\varphi}^{2}\right\rangle\right] .
\end{aligned}
$$

Используя выражения (37), (38), после некоторых упрощений окончательно получим

$$
\begin{aligned}
\left\langle\widehat{T}^{\prime}(X, Y)\right\rangle= & -\frac{1}{2 \sqrt{-G}} \int \frac{1}{\omega} \overline{\left(\psi_{\omega}(\tilde{v}, \lambda) c_{\lambda}\left(q^{\prime}, \tilde{v}\right)\right)} \psi_{\omega}(v, \lambda) \widehat{T}^{l}(X, Y) c_{\lambda}\left(q^{\prime}, v\right) \times \\
& \times d \mu_{0}\left(q^{\prime}\right) d \mu(v) d \mu(\tilde{v}) d \mu(\lambda), \\
\widehat{T}^{l}(X, Y)= & \frac{1}{2}\left\{l_{X}\left(q^{\prime}, \lambda\right), l_{Y}\left(q^{\prime}, \lambda\right)\right\}_{+}+\zeta \mathbf{R i c}(X, Y) \times \\
& +\left(2 \zeta-\frac{1}{2}\right) \mathbf{G}(X, Y) G^{a b} \operatorname{Sp}\left(\left.\operatorname{ad}_{a}\right|_{\mathfrak{m}}\right) l_{b}\left(q^{\prime}, \lambda\right),
\end{aligned}
$$

где через $\{\hat{a}, \hat{b}\}_{+} \equiv \hat{a} \hat{b}+\hat{b} \hat{a}$ обозначен антикоммутатор операторов.

Отдельной задачей является проведение процедуры перенормировки и получение конечных значений вакуумных средних (39), характеризующих эффект поляризации вакуума гравитационным полем. Так как вакуумные средние ТЭИ определяются вычислением функциональных производных по метрике от эффективного действия, данная задача эквивалентна проведению процедуры перенормировки в эффективном действии $W$ квантованного поля.

В рамках метода $\zeta$-регуляризации эффективное действие выражается через обобщенную дзета-функцию оператора уравнения Клейна-Гордона $\widehat{F}=\Delta_{M}+\zeta R+m^{2}$ :

$$
W(s)=-\frac{i}{2}\left(\zeta^{\prime}(s)+\zeta(s) \ln \left(-2 \pi i \mu^{2}\right)\right), \quad W_{\text {ren }}=\left.W(s)\right|_{s=0},
$$

где $\mu$ - нормировочная константа, не зависящая от метрики и имеющая размерность массы. Перенормированные вакуумные средние можно получить, вычисляя 
функциональные производные по метрике от эффективного действия (40), а затем находя аналитическое продолжение при $s=0$ :

$$
\left\langle\widehat{T}_{i j}\right\rangle_{\text {ren }}=\left.\frac{2}{\sqrt{-g_{M}(x)}} \frac{\delta W(s)}{\delta g_{i j}}\right|_{s=0}=-\frac{i}{2}\left(\left.\frac{d}{d s}\right|_{s=0} Z_{i j}(x, t ; s)+Z_{i j}(x, t ; 0) \ln \left(-2 \pi i \mu^{2}\right)\right),
$$

где функция $Z_{i j}(x, t ; s)$ представляет собой аналитическое продолжение по переменной $s$ от вариации дзета-функции $\zeta(s)$ по метрике:

$$
\begin{gathered}
Z_{i j}(x, t ; s)=\frac{2}{\sqrt{-g_{M}(x)}} \frac{\delta \zeta(x, t ; s)}{\delta g^{i j}(x)}=2 s \zeta_{i j}(x, t ; s+1)+s \zeta_{\text {loc }}(s) g_{i j}(x), \\
\zeta_{i j}(x, t ; s) \equiv \int \lambda_{\sigma}^{-s} T_{i j}\left\{\overline{\phi_{\sigma}}, \phi_{\sigma}\right\} d \mu(\sigma) .
\end{gathered}
$$

В работе [23] эта функция носит название дзета-функции ТЭИ. Величины $\zeta_{\text {loc }}(0)$ и $\zeta_{\text {loc }}^{\prime}(0)$ должны быть конечными, и, следовательно,

$$
\lim _{s \rightarrow 0} s \zeta_{\text {loc }}^{\prime}(s)=0, \quad \lim _{s \rightarrow 0} s \zeta_{\text {loc }}(s)=0 .
$$

Тогда для перенормированного ТЭИ можно получить выражение [23]

$$
\begin{aligned}
\left\langle\widehat{T}_{i j}\right\rangle_{\mathrm{ren}}= & i\left(\zeta_{i j}(x, t ; s+1)+\frac{1}{2} g_{i j}(x) \zeta_{\text {loc }}(s)+\right. \\
& \left.+s\left[\zeta_{i j}^{\prime}(x, t ; s+1)+\zeta_{i j}(x, t ; s+1) \ln \left(-2 \pi i \mu^{2}\right)\right]\right)\left.\right|_{s=0} .
\end{aligned}
$$

Такой подход к проблеме получения конечных значений вакуумных средних предложен в работе [23] и применялся в предыдущей работе автора [18] в частном случае, когда однородное пространство является группой Ли с инвариантной метрикой.

Учитывая выражение (34), для квазитетрадных компонент функции $\zeta_{i j}(x, t ; s)$ получим

$$
\begin{gathered}
\zeta(X, Y)(s)=\overline{\zeta(X, Y)}(s)-\zeta \mathbf{R i c}(X, Y) \zeta_{\text {loc }}(s)-\left(2 \zeta-\frac{1}{2}\right) \mathbf{G}(X, Y)\left(\zeta_{\text {loc }}(s-1)+\zeta_{\text {un }}(s)\right), \\
\overline{\zeta(X, Y)}(s)=\int \theta_{\nu \lambda}^{-s} \eta_{\{X} \overline{\phi_{\sigma}(x, t)} \eta_{Y\}} \phi_{\sigma}(x, t) d \mu(\sigma), \\
\zeta_{\text {un }}(s)=2 G^{a b} c_{a} \int \theta_{\nu \lambda}^{-s} \overline{\phi_{\sigma}(x, t)} \eta_{b} \phi_{\sigma}(x, t) d \mu(\sigma) .
\end{gathered}
$$

При помощи обратного преобразования Фурье $(27)$ легко показать, что $\zeta(X, Y)(s)$ не зависит от локальных координат:

$$
\begin{aligned}
\overline{\zeta(X, Y)}(s)= & -\frac{1}{2 \sqrt{-G}} \int \theta_{\nu \lambda}^{-s} \overline{\left(\tilde{\psi}_{\nu}(v, \lambda) c_{\lambda}\left(q^{\prime}, v\right)\right)} \tilde{\psi}_{\nu}(\tilde{v}, \lambda)\left\{l_{X}\left(q^{\prime}, \lambda\right), l_{Y}\left(q^{\prime}, \lambda\right)\right\}_{+} c_{\lambda}\left(q^{\prime}, \tilde{v}\right) \times \\
& \times d \mu_{0}\left(q^{\prime}\right) d \mu(v) d \mu(\tilde{v}) d \mu(\lambda) d \mu(\nu), \\
\zeta_{u n}(s)= & \frac{1}{\sqrt{-G}} G^{a b} c_{a} \int \theta_{\nu \lambda}^{-s} \overline{\left(\tilde{\psi}_{\nu}(v, \lambda) c_{\lambda}\left(q^{\prime}, v\right)\right)} \tilde{\psi}_{\nu}(\tilde{v}, \lambda) l_{b}\left(q^{\prime}, \lambda\right) c_{\lambda}\left(q^{\prime}, \tilde{v}\right) \times \\
& \times d \mu_{0}\left(q^{\prime}\right) d \mu(v) d \mu(\tilde{v}) d \mu(\lambda) d \mu(\nu) .
\end{aligned}
$$


В итоге для квазитетрадных компонент перенормированных вакуумных средних ТЭИ имеем

$$
\begin{aligned}
\left\langle\widehat{T}^{\prime}(X, Y)\right\rangle_{\mathrm{ren}}= & i\left(\zeta(X, Y)(s+1)+\frac{1}{2} \mathbf{G}(X, Y) \zeta_{\mathrm{loc}}(s)+\right. \\
& \left.+s\left[\zeta^{\prime}(X, Y)(s+1)+\zeta(X, Y)(s+1) \ln \left(-2 \pi i \mu^{2}\right)\right]\right)\left.\right|_{s=0} .
\end{aligned}
$$

Таким образом, вычисление перенормированных вакуумных средних (41) сводится к решению редуцированного уравнения (28) и нахождению аналитического продолжения функции $\zeta(X, Y)(s)$.

Можно показать, что если для безмассового скалярного поля в четырехмерном однородном пространстве $M$ отсутствует конформная аномалия ТЭИ, другими словами, $\left(\operatorname{Sp}\left\langle\widehat{T}^{\prime}(X, Y)\right\rangle_{\text {ren }}=0\right)$, то

$$
\lim _{s \rightarrow 0} s \zeta(X, Y)(s+1)=0, \quad \lim _{s \rightarrow 0} s \zeta^{\prime}(X, Y)(s+1)=0
$$

и выражение для перенормированного ТЭИ не зависит от параметра $\mu$.

В качестве примера рассмотрим четырехмерное однородное пространство с пятимерной группой Ли преобразований, алгебра Ли g которой относительно образующих $\left\{e_{0}, \ldots, e_{5}\right\}$ задается коммутационными соотношениями

$$
\left[e_{1}, e_{3}\right]=e_{1}, \quad\left[e_{1}, e_{4}\right]=e_{1}, \quad\left[e_{2}, e_{3}\right]=e_{2}, \quad\left[e_{2}, e_{4}\right]=-e_{2}, \quad\left[e_{0}, e_{A}\right]=0
$$

и подалгеброй изотропии $\mathfrak{h}=\left\{e_{4}\right\}$. Индекс и степень вырождения однородного пространства равны нулю, дефект равен единице.

Алгебра $\mathfrak{g}$ есть разрешимая алгебра Ли индекса 1 , коалгебра $\mathfrak{g}^{*}$ допускает функцию Казимира $K_{0}(f)=f_{0}$ и является объединением непересекающихся инвариантных поверхностей

$$
\begin{aligned}
M_{0}^{( \pm)} & =\left\{ \pm f_{1} f_{2}>0\right\}, \\
M_{1 a}^{( \pm)}=\left\{f_{1}=0, \pm f_{2}>0\right\}, \quad M_{1 b}^{( \pm)} & =\left\{f_{1}=0, \pm f_{2}<0\right\}, \quad M_{2}=\left\{f_{1}=f_{2}=0\right\} .
\end{aligned}
$$

Таким образом, каждая невырожденная К-орбита принадлежит одному из пространств $M_{0}^{( \pm)}$и проходит через параметризованный ковектор $\lambda(\omega)=(\omega, \pm 1, \pm 1,0,0)$, $\omega \in \mathbb{R}$. Для алгебры $\mathfrak{g}$ существует вещественная поляризация $\mathfrak{p}=\left\{e_{0}, e_{1}, e_{2}\right\}$, и $\lambda$-представление, соответствующее невырожденной орбите $\mathcal{O}_{( \pm)}^{0}$, имеет вид

$$
l_{0}=i \omega, \quad l_{1}= \pm i e^{-q_{1}-q_{2}}, \quad l_{2}= \pm i e^{q_{1}-q_{2}}, \quad l_{3}=\partial_{q_{2}}, \quad l_{4}=\partial_{q_{1}},
$$

где $\left(q_{1}, q_{2}\right) \in Q, q_{1} \in[0,2 \pi), q_{2} \in \mathbb{R}$. Операторы (42) косоэрмитовы относительно меры $d \mu_{0}(q)=d q_{1} d q_{2}$.

Однородное пространство допускает трехмерную пуассонову алгебру инвариантных функций $a_{0}=f_{0}, a_{1}=f_{3}, a_{2}=f_{1} \circ f_{2}$ с коммутационными соотношениями $\left\{a_{1}, a_{2}\right\}=-2 a_{2}$ и функцией Казимира $Z(f)=f_{0}$. Операторы $\lambda$-представления $\mathcal{F}$-алгебры инвариантных функций

$$
\hat{a}_{0}=\omega, \quad \hat{a}_{1}=i\left(\partial_{v}-1\right), \quad \hat{a}_{2}=e^{2 v}
$$


эрмитовы относительно меры $d \mu(v)=e^{-2 v} d v, v \in \mathbb{R}$. Решая систему (11), (12), получим $c\left(q^{\prime}, v\right)=\delta\left(q_{2}^{\prime}, v\right)$.

Однородное пространство допускает $G$-инвариантную метрику статического пространства-времени

$$
G^{a b}=\left(\begin{array}{cccc}
1 & 0 & 0 & 0 \\
0 & 0 & -c_{2} & 0 \\
0 & -c_{2} & 0 & 0 \\
0 & 0 & 0 & c_{1}
\end{array}\right), \quad R=6 c_{1}, \quad R_{a b}=-\frac{2 c_{1}}{c_{2}} \delta_{a 2} \delta_{b 3}, \quad c_{1} \geqslant 0, \quad c_{2} \geqslant 0 .
$$

Редуцированное уравнение Клейна-Гордона представляет собой обыкновенное дифференциальное уравнение второго порядка

$$
c_{1}\left(\psi^{\prime \prime}(v)-2 \psi^{\prime}(v)\right)+\left(2 c_{2} e^{2 v}-\omega^{2}+m^{2}+c_{1}\right) \psi(v)=0 .
$$

Функции

$$
\psi_{n}(v)=e^{v} J_{2 n}\left(\sqrt{\frac{2 c_{2}}{c_{1}}} e^{v}\right),
$$

где $J_{\nu}(x)$ - функция Бесселя первого порядка, образуют полный и ортогональный набор решений редуцированного уравнения (43) относительно меры

$$
\int(\cdot) d \mu(n)=4 \sum_{n=1}^{\infty} n(\cdot)
$$

если спектральный параметр удовлетворяет условию $\omega_{n}=4 c_{1} n^{2}+m^{2}$. Функции (44) образуют набор собственных функций оператора (28) с собственными значениями $\theta_{\omega n}=4 c_{1} n^{2}+m^{2}-\omega^{2}$.

Для обобщенной дзета-функции уравнения Клейна-Гордона имеем выражение

$$
\zeta(s)=-\frac{i \sqrt{\pi}}{\sqrt{c_{1}}} \frac{\Gamma(s-1 / 2)}{\Gamma(s)} \sum_{n=1}^{\infty} \frac{\left(4 c_{1} n^{2}+m^{2}\right)^{1 / 2-s}}{4 n^{2}-1} .
$$

Путем несложных вычислений можно показать, что

$$
\zeta_{\text {un }}(s)=0, \quad \overline{\zeta_{00}(s)}=-\frac{1}{2} \frac{\zeta(s-1)}{s-1} .
$$

Тогда для энергии скалярного поля по формуле (41) имеем

$$
\epsilon=\left\langle\widehat{T}_{00}\right\rangle_{0}=-\frac{i}{2} \lim _{s \rightarrow 0} \frac{\zeta(s)}{s} .
$$

Применяя формулу Абеля-Плана для регуляризации дзета-функции (45), получим перенормированное значение плотности энергии скалярного поля

$$
\epsilon=\frac{\pi c_{2}^{2}}{\sqrt{c_{1}}}\left[\frac{m}{2}-2 \int_{m /\left(2 \sqrt{c_{1}}\right)}^{\infty} \frac{\sqrt{4 c_{1} t^{2}-m^{2}} d t}{\left(e^{2 \pi t}-1\right)\left(4 t^{2}-1\right)}\right] .
$$

Выражение (46) описывает эффект поляризации вакуума скалярного поля на однородном пространстве с алгеброй Ли преобразований $\mathfrak{g}$ и подалгеброй изотропии $\mathfrak{h}$. 
Благодарности. Работа выполнена при частичной финансовой поддержке ФЦП "Научные и научно-педагогические кадры инновационной России" (контракты П691, П789), Министерства образования и науки РФ (грант № 14.В37.21.0911 и государственное задание "Наука", контракт № 1.604.2011). Также работа выполнена частично в рамках темы 2.3684.2011 Томского государственного университета.

\section{Список литературы}

[1] А.А. Гриб, С.Г. Мамаев, В. М. Мостепаненко, Квантовые эффекты в интенсивных внешних полях, Атомиздат, М., 1980.

[2] Н. Биррелл, П. Девис, Квантованные поля в искривленном пространстве-времени, Мир, М., 1984.

[3] L. Parker, D. Toms, Quantum Field Theory in Curved Spacetime: Quantized Fields and Gravity, Cambridge Univ. Press, Cambridge, 2009.

[4] J. Hero, Topics in quantum field theory in curved space, arXiv: 1011.4772.

[5] A. DeBenedictis, K.S. Viswanathan, Stress-energy tensors for higher dimensional gravity, arXiv: hep-th/9911060.

[6] S. M. Christensen, Phys. Rev. D, 14:10 (1976), 2490-2501.

[7] Я. Б. Зельдович, А. А. Старобинский, ЖЭТФ, 61:6 (1972), 2161-2175.

[8] V. G. Bagrov, D. M. Gitman, Exact Solutions of Relativistic Wave Equations, Mathematics and its Applications (Soviet Series), 39, Kluwer, Dordrecht, 1990.

[9] E. G. Kalnins, Separation of Variables for Riemannian Spaces of Constant Curvature, Pitman Monographs and Surveys in Pure and Applied Mathematics, 28, Longman Scientific \& Technical, John Wiley \& Sons, New York, 1986.

[10] В.В. Обухов, К.Е. Осетрин, Классификационные проблемы в теории гравитации, Изд-во ТГПУ, Томск, 2007.

[11] А. В. Шаповалов, И. В. Широков, ТМФ, 104:2 (1995), 195-213.

[12] А. В. Шаповалов, И. В. Широков, ТМФ, 106:1 (1996), 3-15.

[13] А. А. Кириллов, 17:4(106) (1962), 57-110.

[14] А. А. Кириллов, Функи. анализ и его прил., 2:2 (1968), 40-55.

[15] А.А. Кириллов, Элементы теории представлений, Наука, М., 1978.

[16] B. Konstant, "Quantzation and Unitary Representations. I. Prequantization", Lectures in Modern Analysis and Applications, III, Lecture Notes in Mathematics, 170, ed. C. T. Taam, Springer, Berlin, 1970, 87-208.

[17] J. M. Souriau, Structure de systèmes dynamique. Maitrises de mathématiques, Dunod, Paris, 1970.

[18] А. И. Бреев, И. В. Широков, А. А. Магазев, ТМФ, 167:1 (2011), 78-95.

[19] И.В. Широков, К-орбиты, гармонический анализ на однородных пространствах и интегрирование дифференииальных уравнений, Препринт, ОмГУ, Омск, 1998.

[20] И. В. Широков, ТМФ, 123:3 (2000), 407-423.

[21] И. В. Широков, ТМФ, 126:3 (2001), 393-408.

[22] С. П. Барановский, И. В. Широков, Сиб. матем. журн., 50:4 (2009), 737-745.

[23] V. Moretti, Phys. Rev. D., 56:12 (1997), 7797-7819.

[24] А. И. Бреев, Изв. вузов. Физика, 53:4 (2010), 86-92.

[25] А. О. Барут, Р. Рончка, Теория представлений групn и ее приложения, т. 1, Мир, М., 1980.

[26] Ю. Д. Бураго, В. А. Залгаллер, Введение в риманову геометрию, Наука, СПб., 1994. 